MALAYA JOURNAL OF MATEMATIK

Malaya J. Mat. 9(04)(2021), 181-193.

http://doi.org/10.26637/mjm904/002

\title{
On rectifying curves in Minkowski 3-space
}

\author{
GÜLŞAH AYDIN ŞEKERCİ*1 ${ }^{*}$, SibEl SEVİNÇ ${ }^{2}$ AND ABDILKADIR CEYLAN ÇÖKEN ${ }^{3}$
}

${ }^{1}$ Faculty of Arts and Sciences, Department of Mathematics, Süleyman Demirel University, Isparta, Turkey.

${ }^{2}$ Faculty of Arts and Sciences, Department of Mathematics, Adnan Menderes University, Aydin, Turkey.

${ }^{3}$ Faculty of Science,Department of Mathematics, Akdeniz University, Antalya, Turkey.

Received 12 July 2021; Accepted 17 September 2021

\begin{abstract}
In this study, we investigate a rectifying curve by using a dilation of a unit speed curve on pseudo-sphere or pseudo-hyperbolic space and its centrode. Firstly, considering a causal character of any curve, we study the connection between Serret-Frenet apparatus of the curve on pseudo-sphere or pseudo-hyperbolic space and its dilation. Then, we extend necessary conditions when the centrode is a rectifying curve. Also, we examine some properties of centrode which is a rectifying curve.
\end{abstract}

AMS Subject Classifications: 53C40, 53C50.

Keywords: Rectifying curve, Minkowski 3-space, Frenet equations, centrode, helix.

\section{Contents}

1 Introduction

3 On the Rectifying Curves and the Dilation of Curves

\section{Introduction}

Characterization of a curve allows to classify curves according to some properties. Thus, instead of doing analysis for each curve, working on these classes is appeared as a more convenient way. One example for the characterized curves is a rectifying curve. The curve was put forward by B. Y. Chen in [1]. In three dimensional Euclidean space $\mathbb{E}^{3}$, if $\alpha: I \subseteq \mathbb{R} \rightarrow \mathbb{E}^{3}$ be a unit speed regular curve with Frenet frame $\{T, N, B\}$ where $T$ is a tangent vector field, $N$ is a normal vector field and $B$ is a binormal vector field, then an osculating plane is a plane spanned by the vector fields $\{T, N\}$, a rectifying plane is a plane spanned by the vector fields $\{T, B\}$ and a normal plane is a plane spanned by the vector fields $\{N, B\}$. The rectifying curve is a curve whose position vector field is located on the rectifying plane. Thus, Chen showed that a rectifying curve $\alpha$ is denoted by the equation

$$
\alpha(s)=\eta(s) T(s)+\xi(s) B(s)
$$

where the functions $\eta$ and $\xi$ are differentiable. Also, it is known that the rectifying curve is characterized as the curve whose ratio of torsion and curvature is a linear fuction of arc parameter in $\mathbb{E}^{3}$ [1]. By analyzing the characterization of rectifying curve, it is interpreted kinematically because the position vector field of the curve

*Corresponding author. Email address: gulsahaydin@sdu.edu.tr (Gülşah AYDIN ŞEKERCI) 


\section{Gülşah AYDIN ŞEKERCİ Sibel SEVINÇ and Abdilkadir Ceylan ÇÖKEN}

states the axis of instantaneous rotation at each point. In addition to that, the connection between rectifying curves and their centrodes is used to study in general mechanics. Firstly, Chen [2] established the connection between rectifying curves and their centrodes in Euclidean 3-space. Since these curves are important, many studies have been conducted on this subject. In this section, we just mention some of them. One of them was made by Ilarslan et. al [5]. According to that, they gave some characterizations of timelike, spacelike, null rectifying curves. Moreover, these curves in dual space were examined in [9]. So, they were explored by taking advantage of the relationship between the curve on a unit dual sphere and the surface theory. Additionally, the modified Darboux vector of curve was described and it was shown that this vector is a rectifying curve [8]. Deshmukh et.al [3] developed the necessary circumstances for the centrode of a curve to be a rectifying curve in Euclidean 3 -space and also, they presented the results about the dilation for rectifying curves and centrodes. This study has been the main motivation of our study.

In this study, we research on the rectifying curves in the Minkowski 3-space. First of all, we use that the dilation of $u(t)$ is written as $\alpha(t)=f(t) u(t)$ where $u(t)$ is a curve on the unit sphere $S^{2}$ centered at the origin and the fuction $f(t)$ is positive differentiable. If the curve $\alpha(t)$ is the rectifying curve, then the dilation factor $f(t)$ is given by $f(t)=a \sec \left(t+t_{0}\right)$. Here, $a>0$ and $t_{0}$ are constants [1]. However, this dilation factor in Minkowski 3-space is defined as different ways for the curves $\alpha(t)$ and $u(t)$ [5]. Considering this difference, Frenet-Serret apparatus of $\alpha$ are given in terms of the curve $u(t)$. It was also shown in [4] that the centrode of $\alpha(t)$ is rectifying curve in Minkowski 3-space when it has non-zero constant curvature and non-constant torsion. Here, we generalize this result. For this, we show that the centrode of any helix is not a rectifying curve in Minkowski 3 -space. Then, using this feature and considering the causal characters of Frenet-Serret vectors, we obtain that the centrode of non-helix curve is the rectifying curve if and only if it satisfies the condition $a \kappa-b \tau=c$ where $a, b, c$ are constants. Finally, there are many cases from the choices of plane and curve, so we consider all the cases and give some notations for the centrode of $\alpha(t)$. We find the relations between Frenet-Serret apparatus of the centrode, which is a rectifying curve, and the Frenet-Serret apparatus of $\alpha$.

\section{Preliminaries}

Let $\mathbb{E}_{1}^{3}$ be a space with the metric $g$ denoted by

$$
g=-d x_{1}^{2}+d x_{2}^{2}+d x_{3}^{2}
$$

where $x=\left(x_{1}, x_{2}, x_{3}\right)$ is a rectangular coordinate system of $\mathbb{E}_{1}^{3}$ and also, it is called the three-dimensional Minkowski space. Pseudo-sphere of radius 1 centered at origin is a hyperquadric in $\mathbb{E}_{1}^{3}$ and is given by

$$
S_{1}^{2}(1)=\left\{v \in \mathbb{E}_{1}^{3} \mid g(v, v)=1\right\}
$$

and pseudo-hyperbolic space of radius 1 centered at origin is defined by

$$
H_{0}^{2}(1)=\left\{v \in \mathbb{E}_{1}^{3} \mid g(v, v)=-1\right\} .
$$

Let $\alpha(s)$ be a curve with an arc-length parameter $s$. It is the non-null curve that satisfies the property $g\left(\alpha^{\prime}(s), \alpha^{\prime}(s)\right)= \pm 1$ where $/$ is the derivation of $\alpha$ [7]. For the Frenet frame $\{T, N, B\}$ of a unit speed non-null curve $\alpha(s)$ in $\mathbb{E}_{1}^{3}$, the Frenet formulas are given in [6] by

$$
\begin{aligned}
T^{\prime}(s) & =\varepsilon_{1} \kappa(s) N(s), \\
N^{\prime}(s) & =-\varepsilon_{0} \kappa(s) T(s)+\varepsilon_{2} \tau(s) B(s), \\
B^{\prime}(s) & =-\varepsilon_{1} \tau(s) N(s)
\end{aligned}
$$

for $g(T, T)=\varepsilon_{0}= \pm 1, g(N, N)=\varepsilon_{1}= \pm 1$ and $g(B, B)=-\varepsilon_{0} \varepsilon_{1}=\varepsilon_{2}$ where $T, N, B$ are known as the tangent vector field, the principal normal vector field, the binormal vector field, respectively. Assume that for 
Frenet frame of $\alpha$, there exists the vector product as

$$
B=T \times N,-\varepsilon_{1} T=N \times B,-\varepsilon_{0} N=B \times T .
$$

The centrode of $\alpha: I \rightarrow \mathbb{E}_{1}^{3}$ is defined by

$$
d=-\varepsilon_{0} \varepsilon_{1} \tau T-\varepsilon_{0} \varepsilon_{1} \kappa B
$$

which is the angular velocity vector of the motion of a mass particle along the curve $\alpha$ and it obeys the laws of motion [4]

$$
T^{\prime}=d \times T, N^{\prime}=d \times N, B^{\prime}=d \times B .
$$

In addition, the rectifying curves are formed by expanding a unit speed curve on a unit sphere with a special factor $f$. To characterize such rectifying curves in $\mathbb{E}_{1}^{3}$, Illarslan et.al [5] gave a theorem. According to this theorem, a unit speed non-null curve $\alpha=\alpha(s)$ is written as the dilation of the curve $u(t)$ to be rectifying curve. So, it is a rectifying curve with a spacelike rectifying plane if and only if it satisfies the condition

$$
\alpha(t)=u(t) \frac{l}{\cos t}
$$

where $l \in \mathbb{R}_{0}^{+}$and $u(t)$ is a unit speed spacelike curve on $S_{1}^{2}(1)$. Also, it is a spacelike (or timelike) rectifying curve with a spacelike (or timelike) position vector if and only if it satisfies the condition

$$
\alpha(t)=u(t) \frac{l}{\sinh t}
$$

where $u(t)$ is a unit speed timelike (or spacelike) curve on $S_{1}^{2}(1)$. Finally, it is a spacelike (or timelike) rectifying curve with a spacelike (or timelike) position vector if and only if it satisfies the condition

$$
\alpha(t)=u(t) \frac{l}{\cosh t}
$$

where $u(t)$ is a unit speed spacelike (or timelike) curve on $H_{0}^{2}(1)$ (or $S_{1}^{2}(1)$ ).

\section{On the Rectifying Curves and the Dilation of Curves}

In Minkowski 3-space, let $u(t)$ be a unit speed spacelike or timelike curve lying on $S_{1}^{2}(1)$ or $H_{1}^{2}(1)$ and $\alpha(t)$ be a dilated rectifying curve of $u(t)$. Now, we give the connections between the Frenet-Serret apparatus of the curves $\alpha(t)$ and $u(t)$ in Minkowski 3-space. For this, the different results are obtained by considering some theorems in [5], because the dilation $\alpha(t)$ of $u(t)$ is defined according to the causal characters of the curves $\alpha(t)$ and $u(t)$.

We assume that $\alpha$ is a spacelike rectifying curve with spacelike position vector on timelike rectifying plane and $u(t)$ is a unit speed timelike curve on pseudo-sphere $S_{1}^{2}(1)$. Then, there exists the dilation $\alpha(t)=$ $\frac{a}{\sinh t} u(t)$. In this case, since the rectifying plane is timelike, clearly its normal vector $N_{\alpha}$ is spacelike, so $g\left(N_{\alpha}, N_{\alpha}\right)=1$. If the curve $\alpha$ is spacelike, the tangent of $\alpha$ is also spacelike. Furthermore, its position vector is spacelike, so we have $g(\alpha, \alpha)>0, g\left(u^{\prime}, u^{\prime}\right)=-1$ and $g(u, u)=1$.

We know that $T_{u}=u^{\prime}$ and $\left\{u, u^{\prime}, u \times u^{\prime}\right\}$ is an orthonormal frame of $\mathbb{E}_{1}^{3}$. We easily write

$$
u^{\prime \prime}=T_{u}^{\prime}=a u+b T_{u}+c u \times T_{u} .
$$

When we use timelike curve $u$ and $\left\langle u^{\prime}, u\right\rangle=0$, we find $a=1$. Similarly, $b=0$ is found. Also, we assume $c=\left\langle u^{\prime \prime}, u \times u^{\prime}\right\rangle=r$. Then, we get

$$
u^{\prime \prime}=u+r u \times u^{\prime} .
$$




\section{Gülşah AYDIN ŞEKERCİ Sibel SEVINÇ and Abdilkadir Ceylan ÇÖKEN}

From (3.1) and using Frenet equations for $u(t)$, there exists

$$
\left\|u^{\prime \prime}\right\|=\sqrt{1+r^{2}}=\kappa_{u}
$$

where $\kappa_{u}$ is a curvature of the unit speed timelike curve $u(t)$ and we find

$$
T_{u}=u^{\prime}, N_{u}=\frac{1}{\kappa_{u}} u+\frac{r}{\kappa_{u}} u \times u^{\prime} .
$$

Theorem 3.1. Let $u(t)$ be a unit speed timelike curve on the unit sphere $S_{1}^{2}(1)$ in $\mathbb{E}_{1}^{3}, \alpha(t)$ be a spacelike rectifying curve on a timelike rectifying plane and its position vector be a spacelike vector defined by $\alpha(t)=$ $\frac{a}{\sinh \left(t+t_{0}\right)} u(t), a \in \mathbb{R}^{+}$. Then, the relation between the Frenet-Serret apparatus of $\alpha(t)$ and $u(t)$ is as follows:

$$
\begin{aligned}
T_{\alpha} & =\sinh \left(t+t_{0}\right) u^{\prime}-\cosh \left(t+t_{0}\right) u \\
N_{\alpha} & =u \times u^{\prime} \\
B_{\alpha} & =\sinh \left(t+t_{0}\right) u-\cosh \left(t+t_{0}\right) u^{\prime} \\
\kappa_{\alpha} & =\frac{1}{a} \sqrt{\kappa_{u}^{2}-1} \sinh ^{3}\left(t+t_{0}\right) \\
\tau_{\alpha} & =\frac{1}{a} \sqrt{\kappa_{u}^{2}-1} \cosh \left(t+t_{0}\right) \sinh ^{2}\left(t+t_{0}\right)
\end{aligned}
$$

where $T_{\alpha}, N_{\alpha}, B_{\alpha}, \kappa_{\alpha}, \tau_{\alpha}$ are the tangent vector, normal vector, binormal vector, curvature, torsion of $\alpha(t)$, respectively, and $\kappa_{u}$ is the curvature of $u(t)$.

Proof. Let $\alpha$ is given by $\alpha(t)=\frac{a}{\sinh \left(t+t_{0}\right)} u(t)$ and $\left\{u, u^{\prime}, u \times u^{\prime}\right\}$ is an orthonormal frame of $\mathbb{E}_{1}^{3}$. Thus, we get $\left\|\alpha^{\prime}(t)\right\|=\frac{a}{\sinh ^{2}\left(t+t_{0}\right)}=v_{\alpha}$ and

$$
T_{\alpha}=\sinh \left(t+t_{0}\right) u^{\prime}-\cosh \left(t+t_{0}\right) u .
$$

Then differentiating $T_{\alpha}$, we obtain

$$
\begin{aligned}
T_{\alpha}^{\prime} & =\cosh \left(t+t_{0}\right) u^{\prime}+\sinh \left(t+t_{0}\right) u^{\prime \prime}-\sinh \left(t+t_{0}\right) u-\cosh \left(t+t_{0}\right) u^{\prime} \\
& =r \sinh \left(t+t_{0}\right) u \times u^{\prime} \\
& =\varepsilon_{1} v_{\alpha} \kappa_{\alpha} N_{\alpha} .
\end{aligned}
$$

From (3.2) we write $r=\sqrt{\kappa_{u}^{2}-1}$. Furthermore, for the left side of (3.2) we say that $u \times u^{\prime}$ is equal to $N_{\alpha}$ because both of them are unit vectors. So, if we write $\left[\frac{r \sinh \left(t+t_{0}\right)}{a} \sinh ^{2}\left(t+t_{0}\right)\right] u \times u^{\prime}=a \kappa_{\alpha} N_{\alpha}$, then we get $N_{\alpha}=u \times u^{\prime}$ and also

$$
\kappa_{\alpha}=\frac{1}{a} \sqrt{\kappa_{u}^{2}-1} \sinh ^{3}\left(t+t_{0}\right)
$$

We know that $B_{\alpha}=T_{\alpha} \times N_{\alpha}$. Then

$$
B_{\alpha}=\sinh \left(t+t_{0}\right) u-\cosh \left(t+t_{0}\right) u^{\prime} .
$$

After differentiating (3.6), we find

$$
\begin{aligned}
B_{\alpha}^{\prime} & =\cosh \left(t+t_{0}\right) u+\sinh \left(t+t_{0}\right) u^{\prime}-\sinh \left(t+t_{0}\right) u^{\prime}-\cosh \left(t+t_{0}\right) u^{\prime \prime} \\
& =-r \cosh \left(t+t_{0}\right) u \times u^{\prime} \\
& =-\varepsilon_{1} v_{\alpha} \tau_{\alpha} N_{\alpha}
\end{aligned}
$$


which gives $\left[r \cosh \left(t+t_{0}\right)\right] u \times u^{\prime}=v_{\alpha} \tau_{\alpha} N_{\alpha}$ that is,

$$
\tau_{\alpha}=\frac{1}{a} \sqrt{\kappa_{u}^{2}-1} \cosh \left(t+t_{0}\right) \sinh ^{2}\left(t+t_{0}\right)
$$

Thus, we establish the desired relationships.

Every curves, which is constituted by $\alpha(t)=\frac{a}{\sinh \left(t+t_{0}\right)} u(t)$, do not have to be a rectifying curve. This result is valid for the curves which are not arc of the great circle. To show that, let $u(t)$ be an arc of the great circle on $S_{1}^{2}(1)$ given by $u(t)=(\sinh t, 0, \cosh t)$. We write $\alpha(t)=a\left(\frac{\sinh t}{\sinh \left(t+t_{0}\right)}, 0, \frac{\cosh t}{\sinh \left(t+t_{0}\right)}\right)$. Hence, we have $\left\|\alpha^{\prime}(t)\right\|=\frac{a}{\sinh ^{2}\left(t+t_{0}\right)}$ and $T_{\alpha}=\left(\sinh t_{0}, 0,-\cosh t_{0}\right)$. Thus, $\kappa_{\alpha}=0$ and finally we say that $\alpha(t)$ is not a rectifying curve.

Theorem 3.2. Let $u(t)$ be a unit speed spacelike curve on the unit sphere $S_{1}^{2}(1)$ in $\mathbb{E}_{1}^{3}$, $\alpha(t)$ be a rectifying curve on a spacelike rectifying plane defined by $\alpha(t)=\frac{a}{\cos \left(t+t_{0}\right)} u(t), a \in \mathbb{R}^{+}$. Then the relation between the Frenet-Serret apparatus of $\alpha(t)$ and $u(t)$ is as follows:

$$
\begin{aligned}
& T_{\alpha}=\cos \left(t+t_{0}\right) u^{\prime}+\sin \left(t+t_{0}\right) u, \\
& N_{\alpha}=u \times u^{\prime}, \\
& B_{\alpha}=-\cos \left(t+t_{0}\right) u+\sin \left(t+t_{0}\right) u^{\prime}, \\
& \kappa_{\alpha}=\frac{1}{a} \sqrt{1-\kappa_{u}^{2}} \cos ^{3}\left(t+t_{0}\right), \\
& \tau_{\alpha}=\frac{-1}{a} \sqrt{1-\kappa_{u}^{2}} \sin \left(t+t_{0}\right) \cos ^{2}\left(t+t_{0}\right)
\end{aligned}
$$

where $T_{\alpha}, N_{\alpha}, B_{\alpha}, \kappa_{\alpha}, \tau_{\alpha}$ are the tangent vector, normal vector, binormal vector, curvature, torsion of $\alpha(t)$, respectively, and $\kappa_{u}$ is the curvature of $u(t)$.

Theorem 3.3. Let $u(t)$ be a unit speed spacelike curve on the unit sphere $S_{1}^{2}(1)$ in $\mathbb{E}_{1}^{3}, \alpha(t)$ be a timelike rectifying curve on a timelike rectifying plane and its position vector be a timelike vector defined by $\alpha(t)=$ $\frac{a}{\sinh \left(t+t_{0}\right)} u(t), a \in \mathbb{R}^{+}$. Then the relation between the Frenet-Serret apparatus of $\alpha(t)$ and $u(t)$ is as follows:

$$
\begin{aligned}
T_{\alpha}= & \frac{\sinh \left(t+t_{0}\right)}{\sqrt{\cosh 2\left(t+t_{0}\right)}} u^{\prime}-\frac{\cosh \left(t+t_{0}\right)}{\sqrt{\cosh 2\left(t+t_{0}\right)}} u \\
N_{\alpha}= & \frac{-2 \sinh \left(t+t_{0}\right)}{f(t) \sqrt{\cosh 2\left(t+t_{0}\right)}}\left(\cosh \left(t+t_{0}\right) u^{\prime}+\sinh \left(t+t_{0}\right) u\right) \\
& -\frac{1}{f(t)} \sqrt{\left(1-\kappa_{u}^{2}\right) \cosh 2\left(t+t_{0}\right)} u \times u^{\prime}, \\
B_{\alpha}= & \frac{\sqrt{1-\kappa_{u}^{2}}}{f(t)}\left\{\sinh \left(t+t_{0}\right) u+\cosh \left(t+t_{0}\right) u^{\prime}\right\}+\frac{2 \sinh \left(t+t_{0}\right)}{f(t)} u \times u^{\prime}, \\
\kappa_{\alpha}= & \frac{\sinh ^{3}\left(t+t_{0}\right)}{a \cosh ^{3 / 2} 2\left(t+t_{0}\right)}\left\{4 \sinh ^{2}\left(t+t_{0}\right)-\left(1-\kappa_{u}\right)^{2} \cosh 2\left(t+t_{0}\right)\right\}^{1 / 2}, \\
\tau_{\alpha}= & \frac{\sqrt{\cosh ^{2}\left(t+t_{0}\right)}}{f^{2}(t)}-2 \kappa_{u} \sinh ^{\prime}\left(t+t_{0}\right)-\sqrt{1-\kappa_{u}^{2}} \cosh \left(t+t_{0}\right)\left(1+\kappa_{u}^{2}\right) \\
& \left.-2 \frac{f^{\prime}(t)}{f(t)} \sqrt{1-\kappa_{u}^{2}} \sinh \left(t+t_{0}\right)\left(1+\sqrt{1-\kappa_{u}^{2}}\right)\right]
\end{aligned}
$$




\section{Gülşah AYDIN ŞEKERCİ Sibel SEVINÇ and Abdilkadir Ceylan ÇÖKEN}

where $T_{\alpha}, N_{\alpha}, B_{\alpha}, \kappa_{\alpha}, \tau_{\alpha}$ are the tangent vector, normal vector, binormal vector, curvature, torsion of $\alpha(t)$, respectively, and $\kappa_{u}$ is the curvature of $u$. Also, $f(t)$ is the function such that $f(t)=\left\{\left(1-\cosh 2\left(t+t_{0}\right)\right)^{2}-\right.$ $\left.\left(1-\kappa_{u}^{2}\right) \cosh 2\left(t+t_{0}\right) \sinh ^{2}\left(t+t_{0}\right)\right\}$.

Theorem 3.4. Let $u(t)$ be a unit speed spacelike curve on the unit sphere $H_{0}^{2}(1)$ in $\mathbb{E}_{1}^{3}$, $\alpha(t)$ be a spacelike rectifying curve on a timelike rectifying plane and its position vector be a timelike vector defined by $\alpha(t)=$ $\frac{a}{\cosh \left(t+t_{0}\right)} u(t)$. Then the relation between the Frenet apparatus of $\alpha(t)$ and $u(t)$ is as follows:

$$
\begin{aligned}
& T_{\alpha}=\cosh \left(t+t_{0}\right) u^{\prime}-\sinh \left(t+t_{0}\right) u, \\
& N_{\alpha}=u \times u^{\prime}, \\
& B_{\alpha}=-\cosh \left(t+t_{0}\right) u+\sinh \left(t+t_{0}\right) u^{\prime}, \\
& \kappa_{\alpha}=\frac{1}{a} \sqrt{1+\kappa_{u}^{2}} \cosh ^{3}\left(t+t_{0}\right), \\
& \tau_{\alpha}=-\frac{1}{a} \sqrt{1+\kappa_{u}^{2}} \sinh \left(t+t_{0}\right) \cosh ^{2}\left(t+t_{0}\right)
\end{aligned}
$$

where $T_{\alpha}, N_{\alpha}, B_{\alpha}, \kappa_{\alpha}, \tau_{\alpha}$ are the tangent vector, normal vector, binormal vector, curvature, torsion of $\alpha(t)$, respectively, and $\kappa_{u}$ is the curvature of $u(t)$.

Theorem 3.5. Let $u(t)$ be a unit speed timelike curve on the unit sphere $S_{1}^{2}(1)$ in $\mathbb{E}_{1}^{3}, \alpha(t)$ be a timelike rectifying curve on a timelike rectifying plane and its position vector be a spacelike vector defined by $\alpha(t)=$ $\frac{a}{\cosh \left(t+t_{0}\right)} u(t)$. Then the relation between the Frenet-Serret apparatus of $\alpha(t)$ and $u(t)$ is as follows:

$$
\begin{aligned}
T_{\alpha} & =\cosh \left(t+t_{0}\right) u^{\prime}-\sinh \left(t+t_{0}\right) u \\
N_{\alpha} & =u \times u^{\prime} \\
B_{\alpha} & =\cosh \left(t+t_{0}\right) u-\sinh \left(t+t_{0}\right) u^{\prime}, \\
\kappa_{\alpha} & =\frac{1}{a} \sqrt{\kappa_{u}^{2}-1} \cosh ^{3}\left(t+t_{0}\right), \\
\tau_{\alpha} & =\frac{1}{a} \sqrt{\kappa_{u}^{2}-1} \sinh \left(t+t_{0}\right) \cosh ^{2}\left(t+t_{0}\right)
\end{aligned}
$$

where $T_{\alpha}, N_{\alpha}, B_{\alpha}, \kappa_{\alpha}, \tau_{\alpha}$ are the tangent vector, normal vector, binormal vector, curvature, torsion of $\alpha(t)$, respectively, and $\kappa_{u}$ is the curvature of $u(t)$.

\section{Centrodes as Rectifying Curves}

In this section, considering the Frenet vectors of the curve $\alpha$ in $\mathbb{E}_{1}^{3}$ and their causal characters, we give a proposition that if the curve $\alpha$ is the helix, then its centrode is a line segment. Then using this proposition, we examine the features, which should be provided by the curves whose centrodes are the rectifying curves except the helix. In the previous studies [1, 4], it was shown for the curves with the constant (or non-constant) curvature $\kappa$ and the non-constant (or constant) torsion $\tau$. Here, this result has been expanded.

Let $\alpha: I \subseteq \mathbb{R} \rightarrow \mathbb{E}_{1}^{3}$ be a unit speed curve with Frenet frame $\left\{T_{\alpha}, N_{\alpha}, B_{\alpha}\right\}$ and also,

$$
g\left(T_{\alpha}, T_{\alpha}\right)=\varepsilon_{0}, g\left(N_{\alpha}, N_{\alpha}\right)=\varepsilon_{1}, g\left(B_{\alpha}, B_{\alpha}\right)=\varepsilon_{2} .
$$

Morever, let $d: I \subseteq \mathbb{R} \rightarrow \mathbb{E}_{1}^{3}$ be the centrode of $\alpha$ with its Frenet frame $\left\{T_{d}, N_{d}, B_{d}\right\}$. Then we have $T_{\alpha}^{\prime}=$ $d \times T_{\alpha}, N_{\alpha}^{\prime}=d \times N_{\alpha}$ and $B_{\alpha}^{\prime}=d \times B_{\alpha}$. For all the cases of the unit speed curve $\alpha$, the centrode $d$ is written by

$$
d=-\varepsilon_{0} \varepsilon_{1} \tau_{\alpha} T_{\alpha}-\varepsilon_{0} \varepsilon_{1} \kappa_{\alpha} B_{\alpha} .
$$




\section{On rectifying curves in Minkowski 3-space}

Now, we find the Serret-Frenet apparatus of $d$. For this, we use $d^{\prime}=-\varepsilon_{0} \varepsilon_{1} \tau_{\alpha}^{\prime} T_{\alpha}-\varepsilon_{0} \varepsilon_{1} \kappa_{\alpha}^{\prime} B_{\alpha}$. Using $d^{\prime}$ we obtain $\left\|d^{\prime}\right\|^{2}=\left(\tau_{\alpha}^{\prime}\right)^{2} \varepsilon_{0}+\varepsilon_{2}\left(\kappa_{\alpha}^{\prime}\right)^{2}$, then the speed of centrode $d$ is given by $v_{d}=\sqrt{\varepsilon_{0}\left(\tau_{\alpha}^{\prime}\right)^{2}+\varepsilon_{2}\left(\kappa_{\alpha}^{\prime}\right)^{2}}$. Additionally,

$$
T_{d}=-\frac{\varepsilon_{0} \varepsilon_{1} \tau_{\alpha}^{\prime} T_{\alpha}}{v_{d}}-\frac{\varepsilon_{0} \varepsilon_{1} \kappa_{\alpha}^{\prime} B_{\alpha}}{v_{d}}
$$

Differentiating (4.3) we find

$$
T_{d}^{\prime}=\left(-\frac{\varepsilon_{0} \varepsilon_{1} \tau_{\alpha}^{\prime}}{v_{d}}\right)^{\prime} T_{\alpha}-\frac{\varepsilon_{0} \varepsilon_{1} \tau_{\alpha}^{\prime}}{v_{d}} T_{\alpha}^{\prime}+\left(-\frac{\varepsilon_{0} \varepsilon_{2} \kappa_{\alpha}^{\prime}}{v_{d}}\right)^{\prime} B_{\alpha}-\frac{\varepsilon_{0} \varepsilon_{2} \kappa_{\alpha}^{\prime}}{v_{d}} B_{\alpha}^{\prime} .
$$

The centrode $d$ does not have to be unit speed, then we get

$$
\begin{aligned}
\varepsilon_{1}^{d} \kappa_{d} v_{d} N_{d} & =\left(-\frac{\varepsilon_{0} \varepsilon_{1} \tau_{\alpha}^{\prime}}{v_{d}}\right)^{\prime} T_{\alpha}-\frac{\varepsilon_{0} \tau_{\alpha}^{\prime}}{v_{d}} \kappa_{\alpha} N_{\alpha}-\left(\frac{\varepsilon_{0} \varepsilon_{1} \kappa_{\alpha}^{\prime}}{v_{d}}\right)^{\prime} B_{\alpha}+\frac{\varepsilon_{0} \kappa_{\alpha}^{\prime}}{v_{d}} \tau_{\alpha} N_{\alpha} \\
& =-\left(\frac{\varepsilon_{0} \varepsilon_{1} \tau_{\alpha}^{\prime}}{v_{d}}\right)^{\prime} T_{\alpha}-\frac{\varepsilon_{0} \tau_{\alpha}^{\prime} \kappa_{\alpha}-\varepsilon_{0} \kappa_{\alpha}^{\prime} \tau_{\alpha}}{v_{d}} N_{\alpha}-\left(\frac{\varepsilon_{0} \varepsilon_{1} \kappa_{\alpha}^{\prime}}{v_{d}}\right)^{\prime} B_{\alpha} .
\end{aligned}
$$

Using this equation we give the following proposition about helices.

Proposition 4.1. Let $\alpha: I \rightarrow \mathbb{E}_{1}^{3}$ be a unit speed curve whose curvature $\kappa_{\alpha}$ and torsion $\tau_{\alpha}$ satisfy $\kappa_{\alpha}>0$ and $\varepsilon_{0}\left(\tau_{\alpha}^{\prime}\right)^{2}+\varepsilon_{2}\left(\kappa_{\alpha}^{\prime}\right)^{2} \neq 0$. Then $\alpha$ is a helix if and only if its centrode $d$ is a line segment.

Proof. We assume that the centrode of $\alpha$ is a line segment, then its curvature is zero, namely $\kappa_{d}=0$. In (4.4), the coefficients of $T_{\alpha}, N_{\alpha}, B_{\alpha}$ are also zero since $T_{\alpha}, N_{\alpha}, B_{\alpha}$ vectors are linearly independent. Then, we write the following:

(i) $\left(\frac{\varepsilon_{0} \varepsilon_{1} \tau_{\alpha}^{\prime}}{v_{d}}\right)^{\prime}=0$

(ii) $\frac{\varepsilon_{0} \tau_{\alpha}^{\prime} \kappa_{\alpha}-\varepsilon_{0} \kappa_{\alpha}^{\prime} \tau_{\alpha}}{v_{d}}=0$,

(iii) $\left(\frac{\varepsilon_{0} \varepsilon_{1} \kappa_{\alpha}^{\prime}}{v_{d}}\right)^{\prime}=0$

For $(i i)$, from $v_{d} \neq 0$ there is $\varepsilon_{0} \tau_{\alpha}^{\prime} \kappa_{\alpha}-\varepsilon_{0} \kappa_{\alpha}^{\prime} \tau_{\alpha}=0$. We know that $\varepsilon_{0} \neq 0$. If we divide both sides of the equation to $\varepsilon_{0}$, then we find $\tau_{\alpha}^{\prime} \kappa_{\alpha}-\kappa_{\alpha}^{\prime} \tau_{\alpha}=0$. Thus, we write that $\frac{\tau_{\alpha}^{\prime}}{\kappa_{\alpha}^{\prime}}=\frac{\tau_{\alpha}}{\kappa_{\alpha}}$ and

$$
\left(\frac{\tau_{\alpha}}{\kappa_{\alpha}}\right)^{\prime}=\frac{\tau_{\alpha}^{\prime} \kappa_{\alpha}-\tau_{\alpha} \kappa_{\alpha}^{\prime}}{\kappa_{\alpha}^{2}}=0 .
$$

Consequently, $\frac{\tau_{\alpha}}{\kappa_{\alpha}}$ is constant and $\alpha$ is a helix. Conversely, if $\alpha$ is a helix, then we write $\tau_{\alpha}=c \kappa_{\alpha}, c \neq 0$. Using (4.4),

$$
\begin{aligned}
\varepsilon_{1}^{d} \kappa_{d} v_{d} N_{d} & =-\left(\frac{\varepsilon_{0} \varepsilon_{1} c \kappa_{\alpha}^{\prime}}{v_{d}}\right)^{\prime} T_{\alpha}-\frac{\varepsilon_{0} c \kappa_{\alpha}^{\prime} \kappa_{\alpha}-\varepsilon_{0} \kappa_{\alpha}^{\prime} c \kappa_{\alpha}}{v_{d}} N_{\alpha}-\left(\frac{\varepsilon_{0} \varepsilon_{1} \kappa_{\alpha}^{\prime}}{v_{d}}\right)^{\prime} B_{\alpha} \\
& =\frac{\left(-\varepsilon_{0} \kappa_{\alpha}^{\prime \prime} v_{d}+\varepsilon_{0} \kappa_{\alpha}^{\prime} v_{d}^{\prime}\right)}{v_{d}^{2}}\left(\varepsilon_{1} c T_{\alpha}+\varepsilon_{1} B_{\alpha}\right) .
\end{aligned}
$$

Now, we investigate the cases in Minkowski 3-space. Firstly, we obtain

$$
\kappa_{d}^{2} v_{d}^{2} g\left(N_{d}, N_{d}\right)=\left(\varepsilon_{0} c^{2}+\varepsilon_{2}\right)\left[\left(\frac{\kappa_{\alpha}^{\prime}}{v_{d}}\right)^{\prime}\right]^{2}
$$




\section{Gülşah AYDIN ŞEKERCİ Sibel SEVINÇ and Abdilkadir Ceylan ÇÖKEN}

and also

$$
\varepsilon_{0}^{d}=\varepsilon_{0}\left(\frac{c \kappa_{\alpha}^{\prime}}{v_{d}}\right)^{2}+\varepsilon_{2}\left(\frac{\kappa_{\alpha}^{\prime}}{v_{d}}\right)^{2}
$$

where $\varepsilon_{0}^{d}$ is the signature of $T_{d}$, that is $g\left(T_{d}, T_{d}\right)=\varepsilon_{0}^{d}$. Here, differentiating both sides, we write

$$
2 \varepsilon_{0} \frac{c \kappa_{\alpha}^{\prime}}{v_{d}}\left(\frac{c \kappa_{\alpha}^{\prime}}{v_{d}}\right)^{\prime}+2 \varepsilon_{2} \frac{\kappa_{\alpha}^{\prime}}{v_{d}}\left(\frac{\kappa_{\alpha}^{\prime}}{v_{d}}\right)^{\prime}=0
$$

Hence, it is $\varepsilon_{0} c^{2}+\varepsilon_{2}=0, \frac{\kappa_{\alpha}^{\prime}}{v_{d}}=0$ or $\left(\frac{\kappa_{\alpha}^{\prime}}{v_{d}}\right)^{\prime}=0$.

(1) For $\varepsilon_{0} c^{2}+\varepsilon_{2}=0$, there exists $\kappa_{d}^{2} v_{d}^{2} g\left(N_{d}, N_{d}\right)=0$. From $g\left(N_{d}, N_{d}\right) \neq 0$ and $v_{d}^{2} \neq 0$, then $\kappa_{d}=0$.

(2) For $\frac{\kappa_{\alpha}^{\prime}}{v_{d}}=0$, clearly $\kappa_{d}=0$.

(3) For $\left(\frac{\kappa_{\alpha}^{\prime}}{v_{d}}\right)^{\prime} 0$, clearly $\kappa_{d}=0$.

Therefore, if $\alpha$ is a helix, then the centrode of $\alpha$ is a line segment.

Theorem 4.2. Let $\alpha$ be a unit speed spacelike (timelike) curve in $\mathbb{E}_{1}^{3}$ with a timelike(spacelike) binormal, $\kappa_{\alpha}, \tau_{\alpha} \neq 0$ and $\left(\tau_{\alpha}^{\prime}\right)^{2}-\left(\kappa_{\alpha}^{\prime}\right)^{2} \neq 0$. If $\alpha$ is not a helix, then the centrode $d$ of $\alpha$ is a rectifying curve if and only if $\kappa_{\alpha}$ and $\tau_{\alpha}$ satisfy the equation $a \kappa_{\alpha}-b \tau_{\alpha}=c$ where $a, b, c$ are constants and they provide the conditions $c \neq 0, a^{2}-b^{2} \neq 0$.

Proof. Let $\alpha=\alpha(t)$ be a unit speed curve in $\mathbb{E}_{1}^{3}$ and $\kappa_{\alpha}, \tau_{\alpha} \neq 0, \varepsilon_{0}\left(\tau_{\alpha}^{\prime}\right)^{2}+\varepsilon_{2}\left(\kappa_{\alpha}^{\prime}\right)^{2} \neq 0$, namely $\alpha$ is not a null curve. If $\alpha$ is not a helix, then we use (4.4) and (4.2). So, we get

$$
\varepsilon_{1}^{d} \kappa_{d} v_{d} g\left(N_{d}(t), d(t)\right)=\varepsilon_{0} \tau_{\alpha}\left(\frac{\tau_{\alpha}^{\prime}}{v_{d}}\right)^{\prime}+\varepsilon_{2} \kappa_{\alpha}\left(\frac{\kappa_{\alpha}^{\prime}}{v_{d}}\right)^{\prime} .
$$

If the centrode $d$ of $\alpha$ is a rectifying curve, the multiplication of the position vector field of $d$ and $N_{d}$ is zero. Since $\alpha$ is not a helix, $d$ is not a line segment and $\kappa_{d} \neq 0$. From $\kappa_{d}>0$ and $v_{d} \neq 0$, we write $g\left(N_{d}, d\right)=0$. Then we find

$$
\varepsilon_{0} \tau_{\alpha}\left(\frac{\tau_{\alpha}^{\prime}}{v_{d}}\right)^{\prime}+\varepsilon_{2} \kappa_{\alpha}\left(\frac{\kappa_{\alpha}^{\prime}}{v_{d}}\right)^{\prime}=0
$$

This equation shows that, if the centrode $d$ of $\alpha$ is rectifying curve, then it satisfies (4.2). Now, we use (4.6) and try to obtain better notation. Let $\alpha$ be a unit speed curve in $\mathbb{E}_{1}^{3}$ with a timelike binormal vector. We have

$$
\tau_{\alpha}\left(\frac{\tau_{\alpha}^{\prime}}{v_{d}}\right)^{\prime}-\kappa_{\alpha}\left(\frac{\kappa_{\alpha}^{\prime}}{v_{d}}\right)^{\prime}=0
$$

and from the hypotesis of the theorem, we take $\left(\tau_{\alpha}^{\prime}\right)^{2}-\left(\kappa_{\alpha}^{\prime}\right)^{2} \neq 0$. Then, it is $\left(\tau_{\alpha}^{\prime}\right)^{2}-\left(\kappa_{\alpha}^{\prime}\right)^{2}>0$ or $\left(\tau_{\alpha}^{\prime}\right)^{2}-$ $\left(\kappa_{\alpha}^{\prime}\right)^{2}<0$.

Case 1 : We assume that $\left(\tau_{\alpha}^{\prime}\right)^{2}-\left(\kappa_{\alpha}^{\prime}\right)^{2}>0$. In this situation, if $\tau_{\alpha}^{\prime}$ is zero, then the condition $\left(\tau_{\alpha}^{\prime}\right)^{2}-\left(\kappa_{\alpha}^{\prime}\right)^{2}>$ 0 is not satisfied. So $\tau_{\alpha}^{\prime} \neq 0$. Then, let $\theta_{1}(t)$ be a function defined by $\sin ^{-1}\left(\frac{\kappa_{\alpha}^{\prime}}{\tau_{\alpha}^{\prime}}\right)$. Using the equations

$$
\begin{aligned}
& \sin \theta_{1}(t)=\frac{\kappa_{\alpha}^{\prime}}{\tau_{\alpha}^{\prime}}, \cos \theta_{1}(t)=\frac{\sqrt{\left(\tau_{\alpha}^{\prime}\right)^{2}-\left(\kappa_{\alpha}^{\prime}\right)^{2}}}{\tau_{\alpha}^{\prime}}, \\
& \tan \theta_{1}(t)=\frac{\kappa_{\alpha}^{\prime}}{\sqrt{\left(\tau_{\alpha}^{\prime}\right)^{2}-\left(\kappa_{\alpha}^{\prime}\right)^{2}}}, \sec \theta_{1}(t)=\frac{\tau_{\alpha}^{\prime}}{\sqrt{\left(\tau_{\alpha}^{\prime}\right)^{2}-\left(\kappa_{\alpha}^{\prime}\right)^{2}}},
\end{aligned}
$$


then we get

$$
\begin{aligned}
& \tau_{\alpha}\left(\sec \theta_{1}(t)\right)^{\prime}-\kappa_{\alpha}\left(\tan \theta_{1}(t)\right)^{\prime}=0 \\
& \theta_{1}^{\prime}(t) \sec \theta_{1}(t)\left[\tau_{\alpha} \tan \theta_{1}(t)-\kappa_{\alpha} \sec \theta_{1}(t)\right]=0 .
\end{aligned}
$$

For $\tau_{\alpha} \tan \theta_{1}(t)-\kappa_{\alpha} \sec \theta_{1}(t)=0$, we find easily that $\tau_{\alpha} \tan \theta_{1}(t)=\kappa_{\alpha} \sec \theta_{1}(t)$. Then

$$
\frac{\tau_{\alpha}}{\kappa_{\alpha}}=\frac{\sec \theta_{1}(t)}{\tan \theta_{1}(t)}=\frac{1}{\sin \theta_{1}(t)}=\csc \theta_{1}(t)=\frac{\tau_{\alpha}^{\prime}}{\kappa_{\alpha}^{\prime}} .
$$

So, $\alpha$ is a helix. But, it is contrast to the hypotesis. For $\sec \theta_{1}(t)=0$, we write $\frac{\tau_{\alpha}^{\prime}}{\sqrt{\left(\tau_{\alpha}^{\prime}\right)^{2}-\left(\kappa_{\alpha}^{\prime}\right)^{2}}}=0$, namely, $\tau_{\alpha}^{\prime}=0$ and this is contrast to the hypotesis, too. For $\theta_{1}^{\prime}(t)=0$, then it is clear that $\theta_{1}(t)=$ constant. Furthermore $\sin \theta_{1}(t)=\frac{\kappa_{\alpha}^{\prime}}{\tau_{\alpha}^{\prime}}=c_{1}$ and $c_{1}$ is a constant. If we choose $\frac{b}{a}=c_{1}$, then there is $a \kappa_{\alpha}^{\prime}-b \tau_{\alpha}^{\prime}=0$. From here, $a \kappa_{\alpha}-b \tau_{\alpha}=c$.

Case 2 : We assume that $\left(\tau_{\alpha}^{\prime}\right)^{2}-\left(\kappa_{\alpha}^{\prime}\right)^{2}<0$. In this situation, $\kappa_{\alpha}^{\prime} \neq 0$. Let $\theta_{2}(t)$ be a function defined by $\sin ^{-1}\left(\frac{\tau_{\alpha}^{\prime}}{\kappa_{\alpha}^{\prime}}\right)$. Using the equations

$$
\begin{aligned}
& \sin \theta_{2}(t)=\frac{\tau_{\alpha}^{\prime}}{\kappa_{\alpha}^{\prime}}, \cos \theta_{2}(t)=\frac{\sqrt{\left(\kappa_{\alpha}^{\prime}\right)^{2}-\left(\tau_{\alpha}^{\prime}\right)^{2}}}{\kappa_{\alpha}^{\prime}}, \\
& \tan \theta_{2}(t)=\frac{\tau_{\alpha}^{\prime}}{\sqrt{\left(\kappa_{\alpha}^{\prime}\right)^{2}-\left(\tau_{\alpha}^{\prime}\right)^{2}}}, \sec \theta_{2}(t)=\frac{\kappa_{\alpha}^{\prime}}{\sqrt{\left(\kappa_{\alpha}^{\prime}\right)^{2}-\left(\tau_{\alpha}^{\prime}\right)^{2}}},
\end{aligned}
$$

we get

$$
\theta_{2}^{\prime}(t) \sec \theta_{2}(t)\left[-\tau_{\alpha} \sec \theta_{2}(t)+\kappa_{\alpha} \tan \theta_{2}(t)\right]=0
$$

Thus, we obtain $a \kappa_{\alpha}-b \tau_{\alpha}=c$. Conversely, if $\kappa_{\alpha}$ and $\tau_{\alpha}$ provides the equation $a \kappa_{\alpha}-b \tau_{\alpha}=c$, then we find $\tau_{\alpha}\left(\frac{\tau_{\alpha}^{\prime}}{v_{d}}\right)^{\prime}-\kappa_{\alpha}\left(\frac{\kappa_{\alpha}^{\prime}}{v_{d}}\right)^{\prime}=0$ from $\left(\frac{\kappa_{\alpha}^{\prime}}{v_{d}}\right)^{\prime}=0$ and $\left(\frac{\tau_{\alpha}^{\prime}}{v_{d}}\right)^{\prime}=0$. Also, the proof is done for timelike curve with spacelike binormal vector, similarly.

Theorem 4.3. Let $\alpha$ be a unit speed spacelike curve in $\mathbb{E}_{1}^{3}$ with a spacelike binormal vector, $\kappa_{\alpha}, \tau_{\alpha} \neq 0$ and $\left(\tau_{\alpha}^{\prime}\right)^{2}+\left(\kappa_{\alpha}^{\prime}\right)^{2} \neq 0$. If $\alpha$ is not a helix, then the centrode $d$ of $\alpha$ is a rectifying curve if and only if $\kappa$ and $\tau$ satisfy the equation $a \kappa_{\alpha}-b \tau_{\alpha}=c$ where $a, b, c$ are constants and they provide the conditions $c \neq 0, a^{2}+b^{2} \neq 0$.

Proof. We assume that $\varepsilon_{0}=1$ and $\varepsilon_{2}=1$. Then, there exists

$$
\tau_{\alpha}\left(\frac{\tau_{\alpha}^{\prime}}{v_{d}}\right)^{\prime}+\kappa_{\alpha}\left(\frac{\kappa_{\alpha}^{\prime}}{v_{d}}\right)^{\prime}=0
$$

and $\left(\tau_{\alpha}^{\prime}\right)^{2}+\left(\kappa_{\alpha}^{\prime}\right)^{2} \neq 0$. Thus, we get $\kappa_{\alpha}^{\prime} \neq 0$ or $\tau_{\alpha}^{\prime} \neq 0$.

Case 1 : We assume that $\kappa_{\alpha}^{\prime} \neq 0$. Let $\gamma_{1}(t)$ be a function given by $\gamma_{1}(t)=\tan ^{-1}\left(\frac{\tau_{\alpha}^{\prime}}{\kappa_{\alpha}^{\prime}}\right)$. Then, using

$$
\sin \left(\gamma_{1}(t)\right)=\frac{\tau_{\alpha}^{\prime}}{\sqrt{\left(\tau_{\alpha}^{\prime}\right)^{2}+\left(\kappa_{\alpha}^{\prime}\right)^{2}}}, \cos \left(\gamma_{1}(t)\right)=\frac{\kappa_{\alpha}^{\prime}}{\sqrt{\left(\tau_{\alpha}^{\prime}\right)^{2}+\left(\kappa_{\alpha}^{\prime}\right)^{2}}}
$$




\section{Gülşah AYDIN ŞEKERCİ Sibel SEVINÇ and Abdilkadir Ceylan ÇÖKEN}

we get

$$
\tau_{\alpha} \cos \left(\gamma_{1}(t)\right) \gamma_{1}^{\prime}(t)-\kappa_{\alpha} \sin \left(\gamma_{1}(t)\right) \gamma_{1}^{\prime}(t)=0 .
$$

Thus, we write $\gamma_{1}^{\prime}(t)=0$ and it is a constant. So, we obtain $a \kappa-b \tau=c$. Since $\frac{\tau_{\alpha}}{\kappa_{\alpha}}$ is non-constant, it is $c \neq 0$ and $a^{2}+b^{2} \neq 0$.

Case 2 : We assume that $\tau_{\alpha}^{\prime} \neq 0$. Let $\gamma_{2}(t)$ is a function given by $\gamma_{2}(t)=\tan ^{-1}\left(\frac{\kappa_{\alpha}^{\prime}}{\tau_{\alpha}^{\prime}}\right)$. Then, $\tan \left(\gamma_{2}(t)\right)=\frac{\kappa_{\alpha}^{\prime}}{\tau_{\alpha}^{\prime}}$ and using

$$
\sin \left(\gamma_{2}(t)\right)=\frac{\kappa_{\alpha}^{\prime}}{\sqrt{\left(\tau_{\alpha}^{\prime}\right)^{2}+\left(\kappa_{\alpha}^{\prime}\right)^{2}}}, \cos \left(\gamma_{2}(t)\right)=\frac{\tau_{\alpha}^{\prime}}{\sqrt{\left(\tau_{\alpha}^{\prime}\right)^{2}+\left(\kappa_{\alpha}^{\prime}\right)^{2}}}
$$

we get

$$
\left(\kappa_{\alpha} \cos \left(\gamma_{2}(t)\right)-\tau_{\alpha} \sin \left(\gamma_{2}(t)\right)\right) \gamma_{2}^{\prime}(t)=0 .
$$

It is easy to show that $a \kappa_{\alpha}-b \tau_{\alpha}=c$.

Now, we find the relations between the Frenet-Serret apparatus of the centrode $d$, which is a rectifying curve, and the Frenet-Serret apparatus of $\alpha$.

Theorem 4.4. Let $\alpha$ be a unit speed spacelike curve in $\mathbb{E}_{1}^{3}$ with a timelike binormal vector and its Serret-Frenet apparatus be $\left\{T_{\alpha}, N_{\alpha}, B_{\alpha}, \kappa_{\alpha}, \tau_{\alpha}\right\}$. The centrode $d(t)$ of $\alpha(t)$ is a rectifying curve.

(1) If $d(t)$ is a spacelike curve, then the Serret-Frenet apparatus $\left\{T_{d}, N_{d}, B_{d}, \kappa_{d}, \tau_{d}\right\}$ of centrode is given by

$$
\begin{aligned}
T_{d} & =\frac{-\delta}{\sqrt{1-\hat{c}^{2}}} T_{\alpha}-\frac{\delta \hat{c}}{\sqrt{1-\hat{c}^{2}}} B_{\alpha}, \\
N_{d} & =N_{\alpha}, \\
B_{d} & =\frac{-\delta}{\sqrt{1-\hat{c}^{2}}} B_{\alpha}-\frac{\delta \hat{c}}{\sqrt{1-\hat{c}^{2}}} T_{\alpha}, \\
\kappa_{d} & =\frac{\hat{c} \tau_{\alpha}-\kappa_{\alpha}}{\tau_{\alpha}^{\prime}\left(1-\hat{c}^{2}\right)}, \\
\tau_{d} & =\frac{\hat{c} \kappa_{\alpha}-\tau_{\alpha}}{\tau_{\alpha}^{\prime}\left(1-\hat{c}^{2}\right)}
\end{aligned}
$$

where $\delta$ is the signature of $\tau_{\alpha}^{\prime}, \hat{c}=\frac{b}{a}$ and $a, b$ is defined as in Theorem 4.2.

(2) If $d(t)$ is a timelike curve, then the Serret-Frenet apparatus $\left\{T_{d}, N_{d}, B_{d}, \kappa_{d}, \tau_{d}\right\}$ of centrode is given by

$$
\begin{aligned}
T_{d} & =\frac{-\delta \bar{c}}{\sqrt{1-\bar{c}^{2}}} T_{\alpha}-\frac{\delta}{\sqrt{1-\bar{c}^{2}}} B_{\alpha}, \\
N_{d} & =N_{\alpha} \\
B_{d} & =\frac{-\delta \bar{c}}{\sqrt{1-\bar{c}^{2}}} B_{\alpha}-\frac{\delta}{\sqrt{1-\bar{c} c^{2}}} T_{\alpha}, \\
\kappa_{d} & =\frac{\tau_{\alpha}-\bar{c} \kappa_{\alpha}}{\kappa_{\alpha}^{\prime}\left(1-\bar{c}^{2}\right)} \\
\tau_{d} & =\frac{\kappa_{\alpha}-\bar{c} \tau_{\alpha}}{\kappa_{\alpha}^{\prime}\left(1-\bar{c}^{2}\right)}
\end{aligned}
$$

where $\delta$ is the signature of $\kappa_{\alpha}^{\prime}, \bar{c}=\frac{a}{b}$ and $a, b$ is defined as in Theorem 4.2. 
Proof. From $a \kappa_{\alpha}^{\prime}-b \tau_{\alpha}^{\prime}=0$, since $d(t)$ is a spacelike rectifying curve and $\tau_{\alpha}^{\prime} \neq 0$, we write $\kappa_{\alpha}^{\prime}=\frac{b}{a} \tau_{\alpha}^{\prime}$. For $v_{d}$, we write

$$
v_{d}=\sqrt{\left|\left(\tau_{\alpha}^{\prime}\right)^{2}-\left(\kappa_{\alpha}^{\prime}\right)^{2}\right|}=\left|\tau_{\alpha}^{\prime}\right| \sqrt{1-\hat{c}^{2}}
$$

where $\hat{c}=\frac{b}{a}$. For the Frenet-Serret apparatus, we give the following:

$$
\begin{aligned}
T_{d} & =-\frac{\varepsilon_{0} \varepsilon_{1} \tau_{\alpha}^{\prime}}{v_{d}} T_{\alpha}-\frac{\varepsilon_{0} \varepsilon_{1} \kappa_{\alpha}^{\prime}}{v_{d}} B_{\alpha}=-\frac{\tau_{\alpha}^{\prime}}{\left|\tau_{\alpha}^{\prime}\right| \sqrt{1-\hat{c}^{2}}} T_{\alpha}-\frac{\hat{c} \tau_{\alpha}^{\prime}}{\left|\tau_{\alpha}^{\prime}\right| \sqrt{1-\hat{c}^{2}}} B_{\alpha} \\
\varepsilon_{1}^{d} \kappa_{d} v_{d} N_{d} & =\left(-\frac{\tau_{\alpha}^{\prime}}{v_{d}}\right)^{\prime} T_{\alpha}-\frac{\tau_{\alpha}^{\prime} \kappa_{\alpha}-\kappa_{\alpha}^{\prime} \tau_{\alpha}}{v_{d}} N_{\alpha}-\left(\frac{\kappa_{\alpha}^{\prime}}{v_{d}}\right)^{\prime} B_{\alpha} .
\end{aligned}
$$

Then we get

$$
\begin{aligned}
& N_{d}=N_{\alpha} \\
& \kappa_{d}=\frac{-\tau_{\alpha}^{\prime} \kappa_{\alpha}+\kappa_{\alpha}^{\prime} \tau_{\alpha}}{\left(\left|\tau_{\alpha}^{\prime}\right| \sqrt{1-\hat{c}^{2}}\right)^{2}}=\frac{\hat{c} \tau_{\alpha}-\kappa_{\alpha}}{\tau_{\alpha}^{\prime}\left(1-\hat{c}^{2}\right)}
\end{aligned}
$$

Finally, we see easily that

$$
\begin{aligned}
B_{d}=T_{d} \times N_{d} & =\frac{-\delta}{\sqrt{1-\hat{c}^{2}}} B_{\alpha}-\frac{\hat{c} \delta}{\sqrt{1-\hat{c}^{2}}} T_{\alpha} \\
-\tau_{d} \varepsilon_{1}^{d} v_{d} N_{d} & =\frac{-\delta}{\sqrt{1-\hat{c}^{2}}}\left(-\varepsilon_{1} \tau_{\alpha} N_{\alpha}\right)-\frac{\hat{c} \delta}{\sqrt{1-\hat{c}^{2}}} \varepsilon_{1} \kappa_{\alpha} N_{\alpha}
\end{aligned}
$$

then we have $\tau_{d}=\frac{\hat{c} \kappa_{\alpha}-\tau_{\alpha}}{\tau_{\alpha}^{\prime}\left(1-\hat{c}^{2}\right)}$.

Theorem 4.5. Let $\alpha$ be a unit speed timelike curve in $\mathbb{E}^{3}$ with a spacelike binormal vector. The centrode $d(t)$ of $\alpha(t)$ is a rectifying curve.

(1) If $d(t)$ is a spacelike curve, then the Serret-Frenet apparatus $\left\{T_{d}, N_{d}, B_{d}, \kappa_{d}, \tau_{d}\right\}$ of centrode is given by

$$
\begin{aligned}
T_{d} & =\frac{\delta \bar{c}}{\sqrt{1-\bar{c}^{2}}} T_{\alpha}+\frac{\delta}{\sqrt{1-\bar{c}^{2}}} B_{\alpha} \\
N_{d} & =N_{\alpha} \\
B_{d} & =\frac{\delta \bar{c}}{\sqrt{1-\bar{c}^{2}}} B_{\alpha}+\frac{\delta}{\sqrt{1-\bar{c}^{2}}} T_{\alpha} \\
\kappa_{d} & =\frac{\bar{c} \kappa_{\alpha}-\tau_{\alpha}}{\kappa_{\alpha}^{\prime}\left(1-\bar{c}^{2}\right)} \\
\tau_{d} & =\frac{\bar{c} \tau_{\alpha}-\kappa_{\alpha}}{\kappa_{\alpha}^{\prime}\left(1-\bar{c}^{2}\right)}
\end{aligned}
$$

where $\delta$ is the signature of $\kappa_{\alpha}^{\prime}, \bar{c}=\frac{a}{b}$ and $a, b$ is defined as in Theorem 4.2. 


\section{Gülşah AYDIN ŞEKERCİ Sibel SEVINÇ and Abdilkadir Ceylan ÇÖKEN}

(2) If $d(t)$ is a timelike curve, then the Serret-Frenet apparatus $\left\{T_{d}, N_{d}, B_{d}, \kappa_{d}, \tau_{d}\right\}$ of centrode is given by

$$
\begin{aligned}
T_{d} & =\frac{\delta}{\sqrt{1-\hat{c}^{2}}} T_{\alpha}+\frac{\delta \hat{c}}{\sqrt{1-\hat{c}^{2}}} B_{\alpha}, \\
N_{d} & =N_{\alpha}, \\
B_{d} & =\frac{\delta}{\sqrt{1-\hat{c}^{2}}} B_{\alpha}+\frac{\delta \hat{c}}{\sqrt{1-\hat{c}^{2}}} T_{\alpha}, \\
\kappa_{d} & =\frac{\kappa_{\alpha}-\hat{c} \tau_{\alpha}}{\tau_{\alpha}^{\prime}\left(1-\hat{c}^{2}\right)}, \\
\tau_{d} & =\frac{\tau_{\alpha}-\hat{c} \kappa_{\alpha}}{\tau_{\alpha}^{\prime}\left(1-\hat{c}^{2}\right)}
\end{aligned}
$$

where $\delta$ is the signature of $\tau_{\alpha}^{\prime}, \hat{c}=\frac{b}{a}$ and $a, b$ is defined as in Theorem 4.2.

Theorem 4.6. Let $\alpha$ be a unit speed spacelike curve in $\mathbb{E}_{1}^{3}$ with a spacelike binormal and the centrode $d(t)$ of $\alpha(t)$ be a rectifying curve.

(1) If $\tau_{\alpha}^{\prime} \neq 0$, then the Frenet-Serret apparatus of centrode is given by

$$
\begin{aligned}
T_{d} & =\frac{\delta}{\sqrt{1+\hat{c}^{2}}} T_{\alpha}+\frac{\delta \hat{c}}{\sqrt{1+\hat{c}^{2}}} B_{\alpha}, \\
N_{d} & =N_{\alpha}, \\
B_{d} & =\frac{\delta}{\sqrt{1+\hat{c}^{2}}} B_{\alpha}-\frac{\delta \hat{c}}{\sqrt{1+\hat{c}^{2}}} T_{\alpha}, \\
\kappa_{d} & =\frac{\kappa_{\alpha}-\hat{c} \tau_{\alpha}}{\tau_{\alpha}^{\prime}\left(1+\hat{c}^{2}\right)}, \\
\tau_{d} & =\frac{\tau_{\alpha}+\kappa_{\alpha} \hat{c}}{\tau_{\alpha}^{\prime}\left(1+\hat{c}^{2}\right)}
\end{aligned}
$$

where $\delta$ is the signature of $\tau_{\alpha}^{\prime}, \hat{c}=\frac{b}{a}$ and $a, b$ is defined as in Theorem 4.3.

(2) If $\kappa_{\alpha}^{\prime} \neq 0$, then the Frenet-Serret apparatus of centrode is given by

$$
\begin{aligned}
T_{d} & =\frac{\delta \bar{c}}{\sqrt{1+\bar{c}^{2}}} T_{\alpha}+\frac{\delta}{\sqrt{1+\bar{c}^{2}}} B_{\alpha} \\
N_{d} & =N_{\alpha} \\
B_{d} & =\frac{\delta \bar{c}}{\sqrt{1+\bar{c}^{2}}} B_{\alpha}-\frac{\delta}{\sqrt{1+\bar{c}^{2}}} T_{\alpha}, \\
\kappa_{d} & =\frac{\bar{c} \kappa_{\alpha}-\tau_{\alpha}}{\kappa_{\alpha}^{\prime}\left(1+\bar{c}^{2}\right)} \\
\tau_{d} & =\frac{\bar{c} \tau_{\alpha}+\kappa_{\alpha}}{\kappa_{\alpha}^{\prime}\left(1+\bar{c}^{2}\right)}
\end{aligned}
$$

where $\delta$ is the signature of $\tau_{\alpha}^{\prime}, \bar{c}=\frac{a}{b}$ and $a, b$ is defined as in Theorem 4.3 .

\section{References}

[1] B.Y. CHEN, When does the position vector of a space curve always lie in its rectifying plane?, Am. Math. Mon., 110(2003), 147-152. 
[2] B.Y. Chen And F. Dillen, Rectifying curves as centrodes and extremal curves, Bulletin of the Inst. Math. Academia Sinica, 33(2005), 77-90.

[3] S. Deshmukh, B.Y. Chen and S.H. Alshammari, On rectifying curves in Euclidean 3-space, Turkish J. Math., 42(2018), 609-620.

[4] K. İlarslan And E. Nesovic, On rectifying curves as centrodes and extremal curves in the Minkowski 3-spaces, Novi Sad J. Math., 37(2007), 53-64.

[5] K. Ílarslan, E. Nesovic and M.P. Torgasev, Some characterizations of rectifying curves in the Minkowski 3-spaces, Novi Sad J. Math., 33(2003), 23-32.

[6] W. Kuhnel, Differential Geometry: Curves-Surfaces-Manifolds, American Mathematical Society, USA, (1999).

[7] B. O’NeIll, Semi Riemann Geometry with Applications to Relativity, Academic Press, London, (1983).

[8] B. Yilmaz, İ. Gök and Y. Yayli, Extended rectifying curves in Minkowski 3-space, Adv. Appl. Clifford Algebras, 26(2016), 861-872.

[9] A. YÜCESAn, N. AYyILdIZ And A.C. ÇöKEn, On rectifying dual space curves, Rev. Mat. Complut., 20(2007), 497-506.

This is an open access article distributed under the Creative Commons Attribution License, which permits unrestricted use, distribution, and reproduction in any medium, provided the original work is properly cited. 